PROCEEDINGS OF THE

AMERICAN MATHEMATICAL SOCIETY

Volume 128, Number 3, Pages 837-843

$\mathrm{S}$ 0002-9939(99)05212-0

Article electronically published on July 28, 1999

\title{
SAMPLING SEQUENCES FOR HARDY SPACES OF THE BALL
}

\author{
XAVIER MASSANEDA AND PASCAL J. THOMAS
}

(Communicated by Steven R. Bell)

\begin{abstract}
We show that a sequence $a:=\left\{a_{k}\right\}_{k}$ in the unit ball of $\mathbb{C}^{n}$ is sampling for the Hardy spaces $H^{p}, 0<p<\infty$, if and only if the admissible accumulation set of $a$ in the unit sphere has full measure. For $p=\infty$ the situation is quite different. While this condition is still sufficient, when $n>1$ (in contrast to the one dimensional situation) there exist sampling sequences for $H^{\infty}$ whose admissible accumulation set has measure 0 . We also consider the sequence $a(\omega)$ obtained by applying to each $a_{k}$ a random rotation, and give a necessary and sufficient condition on $\left\{\left|a_{k}\right|\right\}_{k}$ so that, with probability one, $a(\omega)$ is of sampling for $H^{p}, p<\infty$.
\end{abstract}

\section{$\S 1$. INTRODUCTION}

Let $\mathbb{B}_{n}$ denote the unit ball of $\mathbb{C}^{n}$. Let $S^{n}$ denote the unit sphere and $d \sigma$ its normalized Lebesgue measure. Recall that for any $0<p \leq \infty$ the Hardy space $H^{p}\left(\mathbb{B}_{n}\right)$ is the set of functions $f$ holomorphic in $\mathbb{B}_{n}$ such that

$$
\|f\|_{p}:=\left(\sup _{r<1} \int_{S^{n}}|f(r \zeta)|^{p} d \sigma(\zeta)\right)^{1 / p}<\infty
$$

where the integral is replaced by a supremum in the case $p=\infty$.

Roughly speaking, we would like to say that a sequence $a:=\left\{a_{k}\right\}_{k}$ of the unit ball is sampling for the space $H^{p}$ when the values of any function $f \in H^{p}$, restricted to the sequence, determine the function uniquely, and moreover some inequalities between the $H^{p}$-norm and an appropriate norm on the space of functions on the sequence $a$ hold. In [Th] it was shown that a natural notion of sampling for $H^{p}$ is the following.

Given $\alpha>1$, let

$$
\Gamma_{\alpha}(\zeta):=\left\{z \in \mathbb{B}_{n}:|1-\bar{\zeta} \cdot z|<\frac{\alpha}{2}\left(1-|z|^{2}\right)\right\}
$$

be the admissible approach region with vertex at $\zeta \in S^{n}$ and aperture $\alpha$ (see [Ru1, p. 72] for the properties of these regions).

The admissible maximal function on $S^{n}$ is then defined, for every $\alpha>1$, as

$$
M^{\alpha} f(\zeta):=\sup _{z \in \Gamma_{\alpha}(\zeta)}|f(z)| .
$$

Received by the editors May 4, 1998.

1991 Mathematics Subject Classification. Primary 32A35, 32A30; Secondary 30B20, 30D50.

Both authors were partially supported by a program of the Comunitat de Treball dels Pirineus. The second author was also supported by DGICYT grant PB95-0956-C02-01 and CIRIT grant GRQ94-2014.

(C)1999 American Mathematical Society 
For any $0<p \leq \infty$ and for any $\alpha>1$ we have $\left\|M^{\alpha} f\right\|_{p} \leq C_{p, \alpha}\|f\|_{p}$, where, for functions defined on the unit sphere, $\|\cdot\|_{p}$ stands for the usual norm in the space $L^{p}(d \sigma)([\mathrm{Ru} 1,5.6 .5])$.

Following $[\mathrm{Br}-\mathrm{Ni}-\mathrm{Oy}]$ we also consider the corresponding maximal function associated to the sequence $a$ :

$$
M_{a}^{\alpha}(f)(\zeta):=\sup _{z \in a \cap \Gamma_{\alpha}(\zeta)}|f(z)| .
$$

From the above it follows that $\left\|M_{a}^{\alpha}(f)\right\|_{p} \leq C_{p, \alpha}\|f\|_{p}$.

Definition. A sequence $a$ is called a sampling sequence for $H^{p}$ when there exists $\alpha>1$ and a constant $C>0$ such that $\left\|M_{a}^{\alpha}(f)\right\|_{p} \geq C\|f\|_{p}$ for every $f \in H^{p}$.

In the case where $p=\infty$ this simply says that $\sup _{a}|f| \geq C\|f\|_{\infty}$, and by taking powers of $f$ we see that $\sup _{a}|f|=\|f\|_{\infty}$.

Given a sequence $a$ let

$$
A D_{\alpha}(a)=\left\{\zeta \in S^{n}: \zeta \in \overline{a \cap \Gamma_{\alpha}(\zeta)}\right\}
$$

and define the admissible accumulation set as $A D(a):=\bigcup_{\alpha>1} A D_{\alpha}(a)$.

Brown, Shields and Zeller showed that the condition $\sigma(A D(a))=1$ characterizes the sampling sequences for $H^{\infty}$ of the disk ([Br-Sh-Ze, Th. 3, (iii)-(iv)]). It will be important to keep in mind that $\sigma(A D(a))=1$ if and only if $\sigma_{\alpha}(A D(a))=1$ for some $\alpha>1$ large enough (see [Th]). Recently the second author showed that the same condition is actually necessary and sufficient for $a$ to be of sampling for any $H^{p}$ of the disk, $p<\infty[$ Th, Theorem 1].

In this note we first prove that when $n>1$, the condition $\sigma(A D(a))=1$ also characterizes the sampling sequences for $H^{p}, p<\infty$.

Theorem 1. A sequence $a$ is sampling for $H^{p}, p<\infty$, if and only if $\sigma(A D(a))=$ 1.

In particular, sampling sequences for $H^{p}$ are the same for all values $p<\infty$.

For $p=\infty$ the situation is more complicated.

On the one hand it is clear that if $a$ is sampling for $H^{\infty}$, then necessarily $\bar{a} \cap S^{n}=$ $S^{n}$ (if $a$ avoids an open set $\{\zeta \in S:|1-\zeta \cdot \bar{\eta}|<\delta\}$, any peak function for $\eta$, for instance $f(z)=z \cdot \bar{\eta}$, violates the sampling condition). Although $\bar{a} \cap S^{n}=S^{n}$ is also sufficient for sampling in the ball algebra $A\left(\mathbb{B}_{n}\right)$, for general $H^{\infty}$ functions this is far from being sufficient: there are sequences which are contained in an $H^{\infty}$ zero set such that $\bar{a} \cap S^{n}=S^{n}$ (for example any sequence $a$ with $\sum_{k}\left(1-\left|a_{k}\right|\right)<\infty$ having $S^{n}$ as cluster set).

The proof of Theorem 1 shows that $\sigma(A D(a))=1$ is as well sufficient for $a$ to be sampling for $H^{\infty}$. This condition is far from being necessary.

Definition. A set $E$ in $S^{n}$ is a max-set when $\operatorname{esssup}_{E}|f|=\|f\|_{\infty}$ for all $f \in H^{\infty}$.

It is clear from the definition that if $A D(a)$ contains a max-set, then $a$ must be sampling for $H^{\infty}$. Since there exist max-sets of arbitrarily small measure [Ru2, 13.4] it is possible to construct, for every $\varepsilon>0$, a sampling sequence $a$ with $\sigma(A D(a))<$ $\varepsilon$. This can be pushed a little further:

Theorem 2. If $n>1$, there exist sampling sequences for $H^{\infty}$ with $\sigma(A D(a))=0$.

Several conjectures can be made regarding necessary or sufficient conditions for sampling in $H^{\infty}$, although we have not been able to prove any of them. All the 
attempts to prove any of these conjectures have led us to the well-known Fatou problem on radial behaviour of holomorphic bounded functions in higher dimension (see [Ru1, Chapter 11]).

We also prove a probabilistic result on random sampling sequences for $H^{p}$ with prescribed radii, along the lines of the results in $[\mathrm{Bo}],[\mathrm{Co}]$ and $[\mathrm{Ma}]$.

Consider the probability space $\Omega=\prod_{k=1}^{\infty} \Omega_{k}$, where $\Omega_{k}$ is the unit sphere $S^{n}$ for all $k$. $\mathcal{A}_{k}$ denotes the $\sigma$-algebra of Lebesgue measurable sets on $S^{n}$, and $P_{k}$ denotes the normalized Lebesgue measure $\sigma$ on the sphere. An element of $\Omega$ is denoted by $\omega=\left(\zeta_{1}, \zeta_{2}, \ldots\right)$, where $\zeta_{k} \in S^{n}$. Each $\zeta_{k}: \Omega \longrightarrow S^{n}$ can be viewed as a random variable defined on $S^{n}$, with values on $S^{n}$ as well. To construct the space of probability, one can alternatively take $\Omega_{k}=O(2 n)$, the group of rotations of $\mathbb{C}^{n}, P_{k}$ the Haar measure on $O(2 n)$ and $\mathcal{A}_{k}$ the $\sigma$-algebra of measurable sets with respect to the Haar measure in $O(2 n)$. Then the elements of $\Omega$ are denoted by $\omega=\left(\mathcal{R}_{1}, \mathcal{R}_{2}, \ldots\right)$.

Given a sequence $a$ we consider a sequence of independent and uniformly distributed random variables $\zeta_{k}(\omega)$ in $S^{n}$ (resp. $\mathcal{R}_{k}^{\omega}$ in $O(2 n)$ ) and define the associated random sequence as $a(\omega):=\left\{a_{k}(\omega)\right\}_{k}$, where $a_{k}(\omega)=\left|a_{k}\right| \zeta_{k}(\omega)$ (resp. $\left.a_{k}(\omega)=\mathcal{R}_{k}^{\omega}\left(a_{k}\right)\right)$. Notice that $\left|a_{k}\right|=\left|a_{k}(\omega)\right|$ for all $\omega$ and for all $k$.

Theorem 3. Let a be a sequence in $\mathbb{B}_{n}$.

(a) If $\sum_{k=1}^{\infty}\left(1-\left|a_{k}\right|\right)^{n}=\infty$, then $P(\{\omega: \sigma(A D(a(\omega)))=1\})=1$.

(b) If $\sum_{k=1}^{\infty}\left(1-\left|a_{k}\right|\right)^{n}<\infty$, then $\sigma(A D(a(\omega)))=0$ for all $\omega$.

As a consequence of Theorem 1 we have the following:

Corollary. Let a be a sequence in $\mathbb{B}_{n}$.

(a) If $\sum_{k=1}^{\infty}\left(1-\left|a_{k}\right|\right)^{n}=\infty$, then $P\left(\left\{\omega: a(\omega)\right.\right.$ is sampling for $\left.\left.H^{p}\right\}\right)=1$ for any $p \leq \infty$.

(b) If $\sum_{k=1}^{\infty}\left(1-\left|a_{k}\right|\right)^{n}<\infty$, then $P\left(\left\{\omega: a(\omega)\right.\right.$ is sampling for $\left.\left.H^{p}\right\}\right)=0$ for any $p<\infty$.

Some remarks are in order.

When the generalized Blaschke condition $\sum_{k}\left(1-\left|a_{k}\right|\right)^{n}<\infty$ holds, the sequence is actually almost surely contained in an $H^{p}$ zero set, for all $p<\infty$ [Ma, Theorem 1.2.]. Thus the generalized Blaschke condition distinguishes two sharply contrasting situations: either $a(\omega)$ is almost surely sampling for $H^{p}$ or it is almost surely contained in an $H^{p}$ zero set.

In the unit disk the Blaschke condition on $a$ implies that every $a(\omega)$ is an $H^{p}$ zero sequence, for all $p \leq \infty$. In particular, $a(\omega)$ is never a sequence of sampling for $H^{p}$, even for $p=\infty$. On the other hand, when $n>1$ and $\sum_{k}\left(1-\left|a_{k}\right|\right)^{n}<\infty$ we have $\sigma\left(A D_{\alpha}(a(\omega))\right)=0$ for all $\omega$, but as seen in Theorem 2, this is not enough to deduce that $a(\omega)$ is not sampling for $H^{\infty}\left(\mathbb{B}_{n}\right)$.

As in [Co, Corollary 1] one can also randomize the moduli $\left|a_{k}\right|$ independently of $\left\{\zeta_{k}(\omega)\right\}_{k}$ and show that Theorem 3 also holds for $a_{k}(\omega)=r_{k}(\omega) \zeta_{k}(\omega)$, where the $\zeta_{k}(\omega)$ are as before and $\left\{r_{k}(\omega)\right\}_{k}$ satisfy:

(i) $r_{k}(\omega) \in(0,1)$ for all $k$ and: in case (a) almost surely $\sum_{k}\left(1-r_{k}(\omega)\right)^{n}=\infty$; in case (b) almost surely $\sum_{k}\left(1-r_{k}(\omega)\right)^{n}<\infty$.

(ii) each $r_{k}(\omega)$ is independent of $\left\{\zeta_{k}(\omega)\right\}_{k}$.

In the following three sections we prove respectively Theorems 1, 2 and 3 . 


\section{§2. Proof of TheOREM 1}

A function $f$ defined on $\mathbb{B}_{n}$ is said to have admissible limit at $\zeta \in S^{n}$ when the limit $\lim _{\substack{z \rightarrow \zeta \\ z \in \Gamma_{\alpha}(\zeta)}} f(z)$ exists, is finite and is the same for all $\alpha>1$. The limit is denoted by $f^{*}(\zeta)$.

The proof that $\sigma(A D(a))=1$ implies $a$ sampling for $H^{p}, p \leq \infty$, is essentially due to Brown, Shields and Zeller, and we include it for the sake of completeness.

For $\alpha>1$, every $f \in H^{p}$ has admissible limit at almost every $\zeta$ and $\left\|f^{*}\right\|_{p}=\|f\|_{p}$ $[\mathrm{Ru} 1,5.6 .8]$. Thus for almost every $\zeta \in A D_{\alpha}(a)$,

$$
M_{a}^{\alpha}(f) \geq \lim _{\substack{z \rightarrow \zeta \\ z \in a \cap \Gamma_{\alpha}(\zeta)}}|f(z)|=\left|f^{*}(\zeta)\right|
$$

Hence if $\sigma\left(A D_{\alpha}(a)\right)=1$, then necessarily $\left\|M_{a}^{\alpha}(f)\right\|_{p} \geq\left\|f^{*}\right\|_{p}$.

Let us see now that $\sigma(A D(a))=1$ is also necessary, if $p<\infty$.

Assume $\sigma\left(A D_{\alpha}(a)\right)<1$. By the same argument as in [Br-Sh-Ze], we may assume that there exist a compact $A \subset S^{n}$ and $N \in \mathbb{N}$ such that $\sigma(A)>0$ and $\Gamma_{\alpha}(\zeta) \cap a \subset$ $B(0,1-1 / N)$ for all $\zeta \in A$. We will use the following technical result.

Lemma. For any $m \in \mathbb{N} \backslash\{0\}$ and $p>0$, there exists a positive real function $\psi_{m} \in \mathcal{C}\left(\overline{\mathbb{B}_{n}}\right)$ such that:

(i) $\psi_{m}(z) \leq m$ for all $z \in \overline{\mathbb{B}_{n}}$, and $\psi_{m}(z)=m$ for $z \in A$;

(ii) $\psi_{m}(z) \leq 1$ for $z \in B(0,1-1 / N)$;

(iii) $\sigma\left(\left\{\zeta \notin A: M^{\alpha} \psi_{m}(\zeta) \geq 1\right\}\right) \leq m^{-p}$.

Proof. Let $\varrho(\zeta, \eta)=|1-\bar{\zeta} \cdot \eta|$ denote the non-isotropic pseudodistance on $\overline{\mathbb{B}_{n}}$ and let $\varrho(z, A):=\inf _{\zeta \in A} \varrho(z, \zeta)$. Define

$$
\psi_{m}(z):=|z|^{\mu_{m}} \max \left(m\left(1-\lambda_{m} \varrho(z, A)\right)_{+}, 1 / m\right),
$$

where $\lambda_{m}$ and $\mu_{m}$ are sequences of positive numbers increasing to infinity whose growth will be determined later on.

The property (i) is then clear, and we ensure (ii) by choosing $\mu_{m}$ large enough so that $m(1-1 / N)^{\mu_{m}} \leq 1$.

Let us now prove (iii). Take $\zeta \notin A$ and suppose that there exists $z \in \Gamma_{\alpha}(\zeta)$ such that $\psi_{m} \geq 1$. This implies that $m\left(1-\lambda_{m} \varrho(z, A)\right) \geq 1$; thus $\varrho(z, A) \leq 1 / \lambda_{m}$.

Then, by the triangle inequality for $\varrho^{1 / 2}$,

$$
\varrho(\zeta, A)^{1 / 2} \leq\left(\frac{\alpha}{2}\left(1-|z|^{2}\right)\right)^{1 / 2}+\varrho(z, A)^{1 / 2} \leq(\sqrt{\alpha}+1) \varrho(z, A)^{1 / 2} \leq \frac{(\sqrt{\alpha}+1)}{\sqrt{\lambda_{m}}} .
$$

Since $S^{n} \backslash A$ is an open set of finite measure, we may end the proof by choosing $\lambda_{m}$ large enough so that

$$
\sigma\left(\left\{\zeta \notin A: \varrho(\zeta, A) \leq(\sqrt{\alpha}+1)^{2} / \lambda_{m}\right\}\right) \leq m^{-p}
$$

This Lemma and [Ru2, Theorem 3.5] give us, for any $\varepsilon>0$, a polynomial $P_{m}$ such that $\left|P_{m}\right| \leq \psi_{m}$ on the closed ball, and

$$
\sigma\left(\left\{\zeta \in S^{n}:\left|P_{m}(\zeta)\right|<\psi_{m}(\zeta)-\varepsilon\right\}\right)<\varepsilon
$$


For a given $p>0$, and taking $\varepsilon$ small enough, we use Lemma (i) to obtain the following lower bound:

$$
\int_{S^{n}}\left|P_{m}\right|^{p} d \sigma \geq \int_{A}\left|P_{m}\right|^{p} d \sigma \geq \frac{1}{2} \int_{A} \psi_{m}^{p} d \sigma=\frac{m^{p}}{2} \sigma(A) .
$$

On the other hand, $M_{a}^{\alpha} P_{m}(\zeta) \leq M^{\alpha} \psi_{m}(\zeta)$ for $\zeta \notin A$, and by Lemma (ii) also $M_{a}^{\alpha} P_{m}(\zeta) \leq \sup _{z \in B(0,1-1 / N)} \psi_{m}(z) \leq 1$ for $\zeta \in A$. This and Lemma (iii) yield:

$$
\begin{gathered}
\int_{S^{n}}\left(M_{a}^{\alpha} P_{m}\right)^{p} d \sigma \leq \sigma(A)+\int_{S^{n} \backslash A}\left(M^{\alpha} \psi_{m}\right)^{p} d \sigma \\
\leq \sigma(A)+\int_{\left\{\zeta \notin A: M^{\alpha} \psi_{m}>1\right\}}\left(M^{\alpha} \psi_{m}\right)^{p} d \sigma+\int_{\left\{\zeta \notin A: M^{\alpha} \psi_{m} \leq 1\right\}}\left(M^{\alpha} \psi_{m}\right)^{p} d \sigma \\
\leq \sigma(A)+m^{-p}\left(\sup _{\mathbb{B}_{n}} \psi_{m}\right)^{p}+\sigma\left(S^{n} \backslash A\right) \leq 2 .
\end{gathered}
$$

Since this is bounded independently of $m, a$ cannot be sampling for $H^{p}$. This finishes the proof of Theorem 1.

\section{$\S 3$. Proof of Theorem 2}

Let $\left\{\eta_{k}\right\}_{k}$ be a dense sequence on the sphere, and consider for each $k$ the big circle $C_{\eta_{k}}=\left\{e^{i \theta} \eta_{k}: \theta \in[0,2 \pi)\right\}$. Denote $E=\bigcup_{k} C_{\eta_{k}}$.

Take next a dyadic decomposition of each big circle $C_{\eta_{k}}$ : for any $m \in \mathbb{N}$ consider the intervals

$$
I_{m, j}^{(k)}=\left\{e^{i \theta} \eta_{k} \in S^{n}:(j-1) 2^{-m} \leq \frac{\theta}{2 \pi}<j 2^{-m}\right\}, \quad j=1, \ldots, 2^{m} .
$$

Let $\zeta_{m, j}^{(k)}=e^{i 2 \pi 2^{-m}(j-1 / 2)} \eta_{k}$ denote the center of the subinterval $I_{m, j}^{(k)}$.

Our sequence is defined as $a=\left\{a_{m, j}^{(k)}\right\}_{k, m, j}$, where $a_{m, j}^{(k)}=\left(1-\frac{\alpha_{k}}{2^{m}}\right) \zeta_{m, j}^{(k)}$ and $\left\{\alpha_{k}\right\}_{k}$ is such that $\sum_{k}\left(k \alpha_{k}\right)^{n-1}<\infty$.

Let us see first that $a$ is sampling for $H^{\infty}$. By construction, and according to the theorem of Brown, Shields and Zeller, on each slice $D_{\eta_{k}}=: \eta_{k} \mathbb{D}$ the sequence $\left\{a_{m, j}^{(k)}\right\}_{m, j} \subset D_{\eta_{k}}$ is sampling for $H^{\infty}\left(D_{\eta_{k}}\right)$, since the non-tangential accumulation set is all $C_{\eta_{k}}$. Thus, given $f \in H^{\infty}$, every slice function $f_{\eta_{k}}(\lambda)=f\left(\lambda \eta_{k}\right), \lambda \in \mathbb{D}$, has radial limits $f_{\eta_{k}}^{*} \in L^{\infty}(\mathbb{T})$ satisfying $\left\|f_{\eta_{k}}^{*}\right\|_{L^{\infty}(\mathbb{T})} \leq S_{f}$, where $S_{f}=\sup _{a}|f|$. The maximum principle then yields $|f| \leq S_{f}$ in $\bigcup_{k} D_{\eta_{k}}$, which by the density of $\left\{\eta_{k}\right\}_{k}$ in $S^{n}$ already implies $\|f\|_{\infty} \leq S_{f}$.

It remains to prove that $\sigma(A D(a))=0$. Define

$$
F=\left\{\zeta \in S^{n}: \varrho\left(\zeta, C_{\eta_{k}}\right)<k \alpha_{k} \text { for infinitely many } k\right\}
$$

where $\varrho$ is the non-isotropic pseudodistance defined at the beginning of the proof of the Lemma. Since $\sigma(F) \leq \sum_{k \geq p}\left(k \alpha_{k}\right)^{n-1}$ for all $p \in \mathbb{N}$, we deduce that $\sigma(F)=0$.

On the other hand, for every $\zeta \notin E \cup F$, the quotient $\alpha_{k} / \varrho\left(\zeta, C_{\eta_{k}}\right)$ (which is bounded by $1 / k$ for $k$ big enough) tends to 0 , so $\zeta$ is not approachable within an admissible region by points of $a$. Hence $A D(a)$ is contained in the zero measure set $E \cup F$. 


\section{§4. Proof of Theorem 3}

Proof of (a). It will be enough to show that for some $\alpha>1$

$$
\int_{\Omega} \sigma\left(A D_{\alpha}(a(\omega))\right) d P(\omega)=1 .
$$

Notice that

$$
\begin{aligned}
A D_{\alpha}(a(\omega)) & =\bigcap_{p \in \mathbb{N}} \bigcup_{k \geq p} I_{\alpha}\left(a_{k}(\omega)\right) \\
& =\left\{\zeta \in S: \zeta \in I_{\alpha}\left(a_{k}(\omega)\right) \text { for infinitely many } k\right\},
\end{aligned}
$$

where $I_{\alpha}\left(a_{k}(\omega)\right)=\left\{\zeta \in S^{n}: a_{k}(\omega) \in \Gamma_{\alpha}(\zeta)\right\}$.

Since the random variables $\zeta_{k}(\omega)$ are uniformly distributed one has:

$$
P\left(\left\{\omega: \zeta \in I_{\alpha}\left(a_{k}(\omega)\right)\right\}\right)=\sigma\left(I_{\alpha}\left(a_{k}\right)\right)=C\left(1-\left|a_{k}\right|^{2}\right)^{n}
$$

for some constant $C>0$ depending only on $\alpha$ and the dimension.

Now $\sum_{k} P\left(\left\{\omega: \zeta \in I_{\alpha}\left(a_{k}(\omega)\right)\right\}\right)=\infty$, so the Borel-Cantelli lemma yields:

$$
P\left(\left\{\omega: \zeta \in I_{\alpha}\left(a_{k}(\omega)\right) \text { for infinitely many } k\right\}\right)=1 .
$$

In particular

$$
P\left(\left\{\omega: \zeta \in \bigcup_{k \geq p} I_{\alpha}\left(a_{k}(\omega)\right)\right\}\right)=1
$$

for all $\zeta \in S^{n}$ and all $p \in \mathbb{N}$. Thus

$$
\int_{\Omega} \sigma\left(\bigcup_{k \geq p} I_{\alpha}\left(a_{k}(\omega)\right)\right) d P(\omega)=\int_{S^{n}} \int_{\Omega} \mathbb{1} \bigcup_{k \geq p} I_{\alpha}\left(a_{k}(\omega)\right)(\zeta) d P(\omega) d \sigma(\zeta)=1
$$

for all $p \in \mathbb{N}$. This together with (1) shows that the required equality holds.

Proof of (b). This is immediate from (1) and the fact that $\sigma\left(I_{\alpha}\left(a_{k}(\omega)\right)\right)=$ $C\left(1-\left|a_{k}\right|^{2}\right)^{n}$ for all $\omega$.

\section{ACKNOWLEDGEMENTS}

We would like to thank Joaquim Ortega Cerdà for valuable conversations on the subject.

\section{REFERENCES}

[Br-Ni-Oy] Bruna J. - Nicolau A. - Øyma K., A note on interpolation in the Hardy spaces in the disc, Proc. Amer. Math. Soc. 124 (1996), 1197-1204. MR 96g:30066

[Br-Sh-Ze] Brown L., Shields A., Zeller K., On absolutely convergent exponential sums, Trans. Amer. Math. Soc. 96 (1960), 162-183. MR 26:332

[Bo] Bomash G., A Blaschke-type product and random zero sets for Bergman spaces, Ark. Mat. 30 (1992), 45-60. MR 93g:30047

[Co] Cochran W. G., Random Blaschke products, Trans. Amer. Math. Soc. 332 (1990), 731-755. MR 91c:30061

[It] Itô K., Introduction to probability theory, Cambridge University Press, 1978. MR 86k:60001

[Ma] Massaneda X., Random sequences with prescribed radii in the unit ball, Complex Variables 31 (1996), 193-211. MR 98e:32006

[Rd] Rudowicz R., Random interpolating sequences with probability one, Bull. London Math. Soc. 26 (1994), 160-164. MR 95k:30073

[Ru1] Rudin W., Function theory in the unit ball of $\mathbb{C}^{n}$, Springer Verlag, Berlin, 1980. MR 82i:32002 
[Ru2] Rudin W., New constructions of functions holomorphic in the unit ball of $\mathbb{C}^{n}$, CBMS Regional Conf. Ser. in Math. 63 AMS, Providence, 1986. MR 87f:32013

[Th] Thomas P.J., Sampling sets for Hardy spaces of the disk, Proc. Amer. Math. Soc. 126 (1998), 2927-2932. CMP 98:16

Departament de Matemàtica Aplicada i Anàlisi, Universitat de Barcelona, Gran Via, 585, 08071-BARCELONA, SpAin

E-mail address: xavier@cerber.mat.ub.es

Laboratoire Emile Picard, Université Paul Sabatier, 118 route de Narbonne, 31062 Toulouse Cedex, France

E-mail address: pthomas@cict.fr 